\title{
An exact analytical solution of non-Fourier thermal stress in cylindrical shell under periodic boundary condition
}

\author{
Mohammad Reza Talaee $^{\mathrm{a}^{*}}$, MansoorAlizadeh $^{\mathrm{b}}$ and Sadra Bakhshandeh ${ }^{\mathrm{c}}$
}

${ }^{a}$ Assistant Professor, Department of Railway Engineering, Iran University of Science and Technology(IUST), Tehran, Iran

${ }^{b}$ Assistant Professor, Department of Mechanical Engineering, Iran University of Science and Technology(IUST), Tehran, Iran

${ }^{c}$ B.Sc. graduate, Department of Railway Engineering, Iran University of Science and Technology(IUST), Tehran, Iran

\begin{tabular}{|c|c|}
\hline ARTICLE INFO & A B S T RACT \\
\hline $\begin{array}{l}\text { Article history: } \\
\text { Received March 6, } 2014 \\
\text { Accepted 23 August } 2014 \\
\text { Available online } \\
\text { 24 August } 2014 \\
\text { Keywords: } \\
\text { Non-Fourier Conduction } \\
\text { Analytical Solution } \\
\text { Cylindrical Coordinate } \\
\text { Harmonic Boundary Conditions }\end{array}$ & $\begin{array}{l}\text { This paper presents a perfect analytical solution of the hyperbolic asymmetric heat conduction } \\
\text { equation and the related thermal displacement equation within a long hollow cylinder (plain } \\
\text { strain condition) exposed to a harmonic boundary condition. The material is assumed to be } \\
\text { homogeneous and isotropic with temperature-independent thermal properties. The standard } \\
\text { method of separation of variables is used for solving the problem with time-independent } \\
\text { boundary conditions and the Duhamel integral is used for applying the time-dependency. The } \\
\text { results show the wave behavior of Non-Fourier thermal stresses and higher oscillation } \\
\text { amplitude in comparison with Fourier one. The developed analytic answer can be applied for } \\
\text { modeling cylindrical shell of nuclear rod and can be applied as a benchmark to validate the } \\
\text { other numerical solutions. }\end{array}$ \\
\hline
\end{tabular}

C) 2014 Growing Science Ltd. All rights reserved.

\section{Introduction}

In the classical heat conduction theory, which is based on Fourier's law, heat flux has a linear relation with the temperature gradient and the propagation speed of the thermal wave is estimated infinite. Although the Fourier's law may still be sufficiently accurate for most of engineering problems under regular conditions, but it is now accepted that in situations involving extreme thermal gradients, and/or a nano (even micro) temporal/spatial scale, or temperatures near absolute zero, Fourier's heat diffusion theory becomes inaccurate and the non-Fourier effect becomes more important. With the non-Fourier profiles the thermal displacement and thermal stress are influenced and the modified thermoelasticity must be used. The governing equation for classical thermoelasticity is followed from known Fourier constitutive relation of heat flux which is the linear relation between heat flux vector q to temperature gradient in solids

* Corresponding author

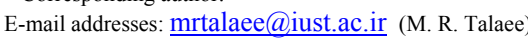


where $\theta$ is temperature and $k$ is the thermal conductivity of material. By implementing Eq. (1) to the energy equation, the parabolic heat conduction equation is derived as:

$k \nabla^{2} \theta=\rho c \frac{\partial \theta}{\partial t}$,

where $\rho c$ is the material heating capacity. The parabolic Eq. (2) estimates an infinite speed for heat propagation. In other worlds, a thermal disturbance on a point of solid body will be senesced immediately on all points. Solving this physically unrealistic phenomenon, attracts too many efforts to itself in recent years. New theories estimate the finite speed and wavy behavior for heat propagation. The hyperbolic equation is based on the constitutive relation of heat flux, which was firstly introduced as: (Cattaneo, 1958; Vernotte, 1958):

$\tau_{\circ} \frac{\partial q}{\partial t}+q=-k(\nabla \theta)$

where $\tau_{0}$ is the relaxation time and it shows that there is a time lag between the imposed thermal gradient and propagated flux. The magnitude of $\tau_{0}$ is about $10^{-10}-10^{-14}$ second for most of the engineering materials. It can be until 20-100 second for non-homogeneous structures and biological tissues (Shen \& Han, 2002). Therefore, the thermal lag is important for some of engineering material. The resulted heat conduction equation in this state is as follows,

$k \nabla^{2} \theta=\rho c\left(\frac{\partial \theta}{\partial t}+\tau_{\circ} \frac{\partial^{2} \theta}{\partial t^{2}}\right)$.

This equation, despite of the Fourier one, is hyperbolic and estimate the wave behavior for heat propagation. So the generalized thermoelasticity with new thermal profiles, must be derived.The generalized thermoelasticity theory in the papers consists of the Lord-Shoulman (LS) and GreenNaghdi (GN) theories (Chandrasekharaiah, 1998). The solution of equations in these models with different coordinates has been derived numerically and in some cases with very simple analytical methods.

For example, Nayfeh (1977) took into consideration the LS model and studied the effects of coupled thermoelastic waves in a one dimensional finite environment analytically. He used the Laplace and Fourier transformations for solving the equations in a semi-infinite model. Using weight function, Nabavi and Shahani (2009) calculated the weight integral analytically and solved the thermal strain equation for a semi-static long cylinder to study the behavior of a crack.

Chen and Lin (1995) applying a combined numerical method of Laplace transformation and control volume scheme studied the problem of transient thermoelasticity with time delay under nonlinear radiation as a boundary condition. The finite element method for the solution of thermoelasticity equation in a finite ambient was used by HosseiniTehrani and Eslami $(2000,2003)$. They studied the effects of the coupler parameter on the propagation of elastics and heat waves.

Chandraskharaiah $(1996,1997)$ studied the wave propagation in a one dimensional semi-infinite sheet with the GN model under sudden temperature exposure of boundaries utilizing the Laplace transformation and found the complete solution of the equations. Taheri et.al. (2004) also solved the coupled thermolelasticity problem in a layer based on the GN model, using the Laplace transformation. Bagri and Eslami (2007) solved the generalized thermoelastic equation in a hollow cylinder and sphere based on LS and GN models, using the Laplace transformation and numerical Inverse Laplace transformation method. 
Two other works have been done which have been used as references for this research. Shahani and Nabavi (2007) solved the Fourier thermoelastic problem in a thick, long and hollow cylinder analytically. In this problem the boundary conditions are time-dependent and applied on the inner surface of the cylinder and after finding temperature profiles, the semi-static thermoelasticity equation was solved and a closed form solution of stresses was presented. Also in the second research work Radu et al. (2008), considered the Fourier heat conduction in a long cylinder with harmonic temperature boundary conditions on the inner surface and solved the semi-static thermoelasticity equation with tension-free boundary conditions, and a closed solution for strains and thermal stresses was offered.

Totally, analytical investigations into the heating problem are fruitful because of reducing the experimental time and cost. Harmonic boundary conditions are commonly encountered boundary conditions in nature and industry such as nuclear rods. The Non-Fourier heat conduction, is important in such cases as very high heat flux, low time of pulse implication and high relaxation time in nonhomogeneous or biological mater. Hence, the non-Fourier theory has found its major use in engineering science fields such as Biomedical Engineering, Electrical Engineering like laser-material interaction, temperature control of superconductors, rapid drying and high heating problems such as in nuclear power plant rode (Atefi \& Talaee, 2011).

In this paper, the hyperbolic heat conduction equation and the thermal stresses in a hollow cylinder are solved analytically under the time dependent boundary temperature. The innovations of the paper are:

- It gives closed and perfect analytical answers for Fourier and Non-Fourier temperature profiles in cylindrical coordinates.

- $\quad$ The method used for solving this paper doesn't need any numerical solutions un-like the Laplace transformation for its inverse integral.

- It gives analytical solutions for thermal stresses and displacement equation and comparing Fourier and Non-Fourier stresses.

- $\quad$ The result can be used for approximately modeling the shielding of nuclear rod with periodic boundary temperature.

- $\quad$ The result can be used as a verification branch of numerical solutions.

\section{Mathematical modeling}

The hyperbolic heat conduction equation in cylindrical coordinates for a long hollow cylinder with inner and outer radius $r_{i}$ and $r_{o}$ is:

$\tau_{\circ} \frac{\partial^{2} \theta}{\partial t^{2}}+\frac{\partial \theta}{\partial t}=\alpha\left[\frac{\partial^{2} \theta}{\partial r^{2}}+\frac{1}{r} \frac{\partial \theta}{\partial r}\right]$

where $\alpha$ is the heat penetration coefficient. The boundary conditions of the problem are considered as follow:

$\left\{\begin{array}{l}\theta\left(r_{i}, t\right)=\theta_{0} \operatorname{Sin}(2 \pi f t) \\ \theta\left(r_{o}, t\right)=0\end{array}\right.$

and the initial conditions are:

$\left\{\begin{array}{l}\theta(r, 0)=0 \\ \left.\frac{\partial \theta}{\partial t}\right|_{r, 0}=0\end{array}\right.$ 
where $f$ is the frequency of temperature boundary changes. Assuming plane strain conditions, which is a reasonable supposition for long cylinders, the coupled displacement-temperature equation in the cylinder is as follows,

$\frac{\partial^{2} u}{\partial r^{2}}+\frac{1}{r} \frac{\partial u}{\partial r}-\frac{u}{r^{2}}-\beta \frac{\partial \theta}{\partial r}=\gamma^{2} \frac{\partial^{2} u}{\partial t^{2}}$

where $\beta=\frac{(1+v)}{(1-v)} a$ and $\gamma^{2}=\frac{\rho(1-2 v)}{2 \mu(1-v)}$ and $a$ is the thermal expansion coefficient, $v$ is the Poisson's ratio, $\mu$ is the cutting module and $\rho$ is the density of the material of the cylinder. Eq. (8) along with Eq. (5) creates a series of thermo elastic non-Fourier equations. Here the answer of Eq. (8) has been studied for a hollow cylinder in a semi-static condition in which the second derivatives of displacement i.e. $\frac{\partial^{2} u}{\partial t^{2}}$ is ignored. Non-zero stresses in the cylinder which is depend on displacement and temperature and are expressed as: (Nabavi \& Shahani, 2009)

$$
\left\{\begin{array}{l}
\sigma_{r}=\frac{2 \mu}{1-2 v}\left[(1-v) \frac{\partial u}{\partial r}+v \frac{u}{r}-a(1+v) \theta\right] \\
\sigma_{\theta}=\frac{2 \mu}{1-2 v}\left[(1-v) \frac{u}{r}+v \frac{\partial u}{\partial r}-a(1+v) \theta\right]
\end{array}\right.
$$

where $\sigma_{r}$ is the radial stress and $\sigma_{\theta}$ is the hoop stress in plane strain condition within the considered cylinder. The inner layer (area) of the cylinder has been affected by an inner pressure of $\mathrm{P}$ while the outer area is fixed, so the stress and displacement boundary conditions are:

$$
\left\{\begin{array}{c}
\sigma_{r}\left(r_{i}, t\right)=-P, \\
u\left(r_{o}, t\right)=0 .
\end{array}\right.
$$

\section{Solution of the temperature equation}

An exact solution of the conduction Eq. (5) with periodic boundary Eqs. (6) and initial Eqs. (7) can take place in the following 4 phases using the separation of variables method and Duhamel Integration (Atefi \& Talaee, 2011; Talaee \& Atefi, 2011).

- $\quad$ Solving the steady equation with non-homogeneous time-independent boundary conditions.

- Solving the transient equation with homogeneous boundary conditions and modified initial conditions.

- Determining the solution of the problem with time-independent boundary conditions by superposition of the two solutions mentioned above.

- Applying the time dependency of boundary conditions within the acquired result, using the Duhamel integration

In continuation, the four above mentioned phases in determining thermal result are expressed.

\subsection{Solution of the steady equation}

First, the steady problem can be rewritten independent of time and with non-homogeneous boundary conditions as following relations:

$$
\frac{\partial^{2} \theta_{1}}{\partial r^{2}}+\frac{1}{r} \frac{\partial \theta_{1}}{\partial r}=0
$$


$\left\{\begin{array}{l}\theta_{1}\left(r_{i}, t\right)=\theta_{0} \\ \theta_{1}\left(r_{o}, t\right)=0\end{array}\right.$

Considering the boundary conditions, the solution is:

$\theta_{1}=\theta_{0}\left[\frac{\ln \frac{r}{r_{o}}}{\ln \frac{r_{i}}{r_{o}}}\right]$

\subsection{Solution of transient equation}

Within the homogeneous boundary condition and modified initial condition, the transient problem in this phase turns into the following form:

$\tau_{\circ} \frac{\partial^{2} \theta_{2}}{\partial t^{2}}+\frac{\partial \theta_{2}}{\partial t}=\alpha\left[\frac{\partial^{2} \theta_{2}}{\partial r^{2}}+\frac{1}{r} \frac{\partial \theta_{2}}{\partial r}\right]$

$\left\{\begin{array}{l}\theta_{2}\left(r_{i}, t\right)=0 \\ \theta_{2}\left(r_{o}, t\right)=0\end{array}\right.$
$\left\{\begin{array}{l}\theta_{2}(r, 0)=-\theta_{1} \\ \left.\frac{\partial \theta_{2}}{\partial t}\right|_{r, 0}=0\end{array}\right.$

Utilizing the separation of variables method and applying the $\theta_{2}(r, t)=R(r) T(t)$ within Eq. (14) and considering the boundary Eqs. (15), the solution of the problem turns into:

$\theta_{2}(r, t)=\sum_{n=1}^{\infty} e^{-\left(\frac{t}{2 \tau_{0}}\right)}\left(E_{n} \operatorname{Cos} \Gamma_{n} t+F_{n} \operatorname{Sin} \Gamma_{n} t\right) \Phi_{0}\left(\omega_{n} r\right)$

where $_{\Phi_{0}}\left(\omega_{n} r\right)=Y_{0}\left(\omega_{n} r\right)-\frac{Y_{0}\left(\omega_{n} r_{o}\right)}{J_{0}\left(\omega_{n} r_{o}\right)} J_{0}\left(\omega_{n} r\right), \Gamma_{n}=\frac{\sqrt{4 \alpha \tau_{o} \omega_{n}^{2}-1}}{2 \tau_{\circ}}$ and $\omega_{n}$ are the roots of the following equation:

$J_{0}\left(\omega r_{o}\right) Y_{0}\left(\omega r_{i}\right)-J_{0}\left(\omega r_{i}\right) Y_{0}\left(\omega r_{o}\right)=0$,

where $\mathrm{J}_{0}$ and $\mathrm{Y}_{0}$ are the Bessel functions of zero degree of type I and II. Using the initial condition of the problem i.e. equations (16), the coefficients $E_{m n}, F_{m n}$ are determined as:

$\left\{\begin{array}{l}E_{n}=-\frac{1}{\delta_{n}} \frac{\theta_{0}}{\ln \frac{r_{i}}{r_{o}} \int_{r_{i}}^{r_{o}} \ln \frac{r}{r_{o}} \Phi_{0}\left(\omega_{n} r\right) r d r,} \\ F_{n}=\frac{1}{2 \tau_{0} \Gamma_{n}} E_{n}\end{array}\right.$

where $\delta_{n}=\int_{r_{i}}^{r_{0}} \Phi_{0}^{2}\left(\omega_{n} r\right) r d r$ and all the above integrals can be performed easily.

\subsection{Superposition of the two solutions}

Based on superposition principle, the general solution of problem (5) under time-independent boundary conditions is equal to the sum of the steady and transient solutions mentioned above: 
$\theta(r, t)=\theta_{1}(r)+\theta_{2}(r, t)=\theta_{0}\left[\frac{\ln \frac{r}{r_{o}}}{\ln \frac{r_{i}}{r_{o}}}\right]+\sum_{n=1}^{\infty} E_{n} G_{n}(t) \Phi_{0}\left(\omega_{n} r\right)$

where $G_{n}(t)$ is equal to:

$G_{n}(t)=e^{-\left(\frac{t}{2 \tau_{0}}\right)}\left(\operatorname{Cos} \Gamma_{n} t+\frac{1}{2 \tau_{0} \Gamma_{n}} \operatorname{Sin} \Gamma_{n} t\right)$

\subsection{Applying time-dependent boundary conditions}

In order to apply the time dependency of boundary conditions (i.e. $f(t)=\operatorname{Sin}(2 \pi f t)$ ), the Duhamel integral is used as (Talaee \& Atefi, 2011):

$\bar{\theta}(r, t)=f(0) \cdot \theta(r, t)+\int_{0}^{t} \theta(r, t-\tau) \frac{\partial f(\tau)}{\partial \tau} d \tau$

Implying Eqs. (20-21) in Eq. (22), the complete solution of the non-Fourier temperature profile within a long cylinder under a periodic boundary condition turns into:

$\bar{\theta}(r, t)=\theta_{0}\left[\frac{\ln \frac{r}{r_{o}}}{\ln \frac{r_{i}}{r_{o}}}\right] \operatorname{Sin}(2 \pi f t)+\sum_{n=1}^{\infty}\left\{\int_{\tau=0}^{t} E_{n}\left(G_{n}(t-\tau) 2 \pi f \cdot \operatorname{Cos}(2 \pi \tau)\right) d \tau\right\} \Phi_{0}\left(\omega_{n} r\right)$

In the same way, the Fourier temperature profiles of the above problem can be derived as:

$\bar{\theta}(r, t)=\theta_{0}\left[\frac{\ln \frac{r}{r_{o}}}{\ln \frac{r_{i}}{r_{o}}}\right] \operatorname{Sin}(2 \pi f t)+\sum_{n=1}^{\infty}\left\{\int_{\tau=0}^{t} E_{n}\left(e^{-\alpha \omega_{n}^{2}(t-\tau)} 2 \pi f \cdot \operatorname{Cos}(2 \pi f \tau)\right) d \tau\right\} \Phi_{0}\left(\omega_{n} r\right)$

\section{Solution of the displacement equation}

Under semi-static condition, the Eq. (8) which is the displacement equation governing the problem becomes as follows: (Shahani \& Nabavi, 2007)

$\left[\frac{\partial}{\partial r}\left(\frac{1}{r} \frac{\partial(r u)}{\partial r}\right)\right]=\frac{(1+v)}{(1-v)} a \frac{\partial \theta}{\partial r}$

and the answer can be shown as:

$u(r, t)=\frac{\beta}{r} \int r \theta(r, t) d r+A(t) r+\frac{B(t)}{r}$

Putting the non-Fourier temperature profile of Eq. (23), in the above equation, the corresponding displacement equation with non-Fourier condition is derived as:

$u(r, t)=A(t) r+\frac{B(t)}{r}+\frac{\beta}{r}\left[\theta_{0} r^{2}\left(\frac{\frac{1}{2} \ln \frac{r}{r_{o}}-\frac{1}{4}}{\ln \frac{r_{i}}{r_{o}}}\right) \operatorname{Sin}(2 \pi f t)+\sum_{n=1}^{\infty}\left\{\int_{\tau=0}^{t} E_{n}\left(G_{n}(t-\tau) 2 \pi f \cdot \operatorname{Cos}(2 \pi f \tau) d \tau\right\} \frac{r}{\omega_{n}} \Phi_{1}\left(\omega_{n} r\right)\right]\right.$

where $\Phi_{1}\left(\omega_{n} r\right)=Y_{1}\left(\omega_{n} r\right)-\frac{Y_{0}\left(\omega_{n} r_{o}\right)}{J_{0}\left(\omega_{n} r_{o}\right)} J_{1}\left(\omega_{n} r\right)$ and $J_{1}$ and $Y_{1}$ are the Bessel functions of first order of type I and II respectively. Coefficients of $A(t)$ and $B(t)$ are determined using Eq. (9) and Eq. (10) as: 
$A(t)=-\frac{B(t)}{r_{o}^{2}}+\beta\left[\frac{1}{4 \ln \frac{r_{i}}{r_{o}}} \operatorname{Sin}(2 \pi f t)-\sum_{n=1}^{\infty}\left\{\int_{\tau=0}^{t} E_{n}\left(G_{n}(t-\tau) 2 \pi f \tau \operatorname{Cos}(2 \pi f \tau) d \tau\right)\right\} \frac{1}{r_{o} \omega_{n}} \Phi_{1}\left(\omega_{n} r_{o}\right)\right]$

$B(t)=\frac{r_{i}^{2} r_{o}^{2}}{(1-2 v) r_{o}^{2}+r_{i}^{2}}\left[\left(\frac{\beta}{2}+(v-1) \frac{\beta}{2 \ln \frac{r_{i}}{r_{o}}}-a(1+v)\right) \theta_{0} \sin (2 \pi f t)+\right.$

$\left.\sum_{n=1}^{\infty}\left\{\int_{\tau=0}^{t} E_{n}\left(G_{n}(t-\tau) 2 \pi f \tau \operatorname{Cos}(2 \pi f \tau) d \tau\right)\right\}[(1-v) \beta-a(1+v)] \Phi_{0}\left(\omega_{n} r_{i}\right)+\left[(1+2 v) \frac{\beta}{\omega_{n} r_{i}} \Phi_{1}\left(\omega_{n} r_{i}\right)+\frac{\beta}{\omega_{n} r_{0}} \Phi_{1}\left(\omega_{n} r_{0}\right)\right]+\frac{P(1-2 v)}{2 \mu}\right]$

In the same way, the analytical displacement relation with Fourier profiles could be determined as:

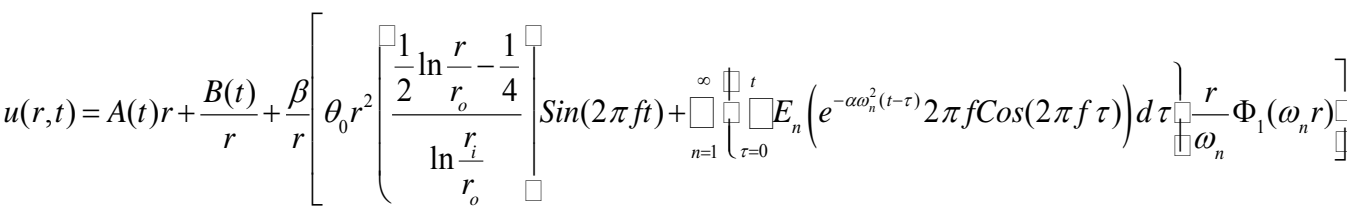

The coefficient $\mathrm{A}(\mathrm{t})$ and $\mathrm{B}(\mathrm{t})$ in Eq. (30)are determined for the Fourier condition too, in the following fashion after simplifications:

$$
\begin{aligned}
& A(t)=-\frac{B(t)}{r_{o}^{2}}+\beta\left[\frac{1}{4 \ln \frac{r_{i}}{r_{0}}} \operatorname{Sin}(2 \pi f t)-\sum_{n=1}^{\infty}\left\{\int_{\tau=0}^{t} E_{n}\left(e^{-\alpha \omega_{n}^{2}(t-\tau)} 2 \pi f \tau \operatorname{Cos}(2 \pi f \tau) d \tau\right)\right\} \frac{1}{r_{0} \omega_{n}} \Phi_{1}\left(r_{0} \omega_{n}\right)\right] \\
& B(t)=\frac{r_{i}^{2} r_{o}^{2}}{(1-2 v) r_{o}^{2}+r_{i}^{2}}\left[\left(\frac{\beta}{2}+(v-1) \frac{\beta}{2 \ln \frac{r_{i}}{r_{o}}}-a(1+v)\right] \theta_{0} \operatorname{Sin}(2 \pi f t)+\sum_{n=1}^{\infty}\left\{\int_{\tau=0}^{t} E_{n}\left(e^{-\alpha \omega_{i}^{2}(t-\tau)} 2 \pi f \tau \operatorname{Cos}(2 \pi f \tau) d \tau\right)\right\}[(1-v) \beta-a(1+v)] \Phi_{0}\left(\omega_{n} r_{i}\right)\right. \\
& \left.+\left[(1+2 v) \frac{\beta}{r_{i} \omega_{n}} \Phi_{1}\left(\omega_{n} r_{i}\right)+\frac{\beta}{r_{o} \omega_{n}} \Phi_{1}\left(\omega_{n} r_{o}\right)\right]+\frac{P(1-2 v)}{2 \mu}\right] .
\end{aligned}
$$

With the determination of the displacement equations such as Eq. (27) and Eq. (30), the nonzero thermal stresses within the cylinder (radial and hoop stresses) can be determined according to Eq. (9). The below dimensionless parameters are considered for plotting temperature and stresses profiles:

$\Theta=\frac{\bar{\theta}}{\theta_{0}}, F o=\frac{\alpha t}{r_{o}^{2}}, V e=\sqrt{\frac{\alpha \tau_{o}}{r_{o}^{2}}}, r^{\prime}=\frac{r}{r_{o}}$.

The magnitude of temperature profile and thermal stresses have been plotted within dimensionless figures under time-dependent boundary temperature of the cylinder shell with frequencies of $f=0.1$ and 0.5 . In this modeling the inner and outer radius (of the cylinder) are considered to be equal to $r_{i}=0.5, r_{0}=1$ and the torsion elasticity module is $\mu=80(G P a)$, while Poisson coefficient, thermal expansion coefficient and the internal pressure of the cylinder are considered as $v=0.3, a=12 \times 10^{-6}(1 / C)$ and $P_{i}=100(\mathrm{MPa})$, respectively. The magnitudes considered here, have been acquired for nuclear fuel rod are as mentioned in article (Radu et al., 2008). 


\section{Results}

Comparing dimensionless Fourier and non-Fourier profiles have been plotted along with radius in Figs. 1 and Fig. 3. Careful observation in Figs. 1-3 shows that non-Fourier temperature profiles unlike Fourier profiles have delay in their diffusion through the thickness of the cylinder. That means there is a time lag till the temperature gradient - caused by imposing the boundary temperature of the inner layer - is diffused into the entire thickness of the cylinder wall. In addition, due to the wavy nature of non-Fourier profiles, the effect of increasing frequency of boundary temperature can be seen in the non-Fourier profiles because of the inertial behavior, there would be a superimposition of thermal waves and thus their amplitude is increased compared with the Fourier one.

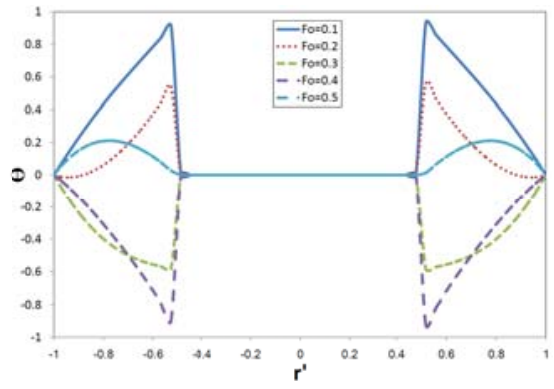

Fig. 1. Temperature profile for Fourier condition in various times with excitation frequency function of 0.1

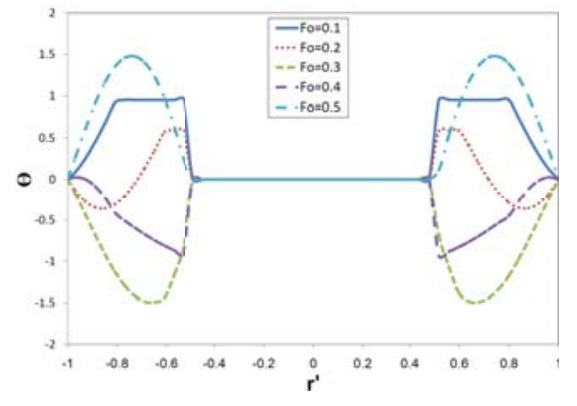

Fig. 2. Temperature profile for non-Fourier condition in various times with excitation frequencies function of 0.1

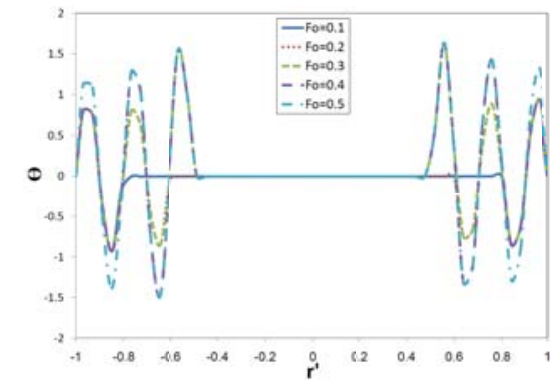

Fig. 3. Non-Fourier temperature profiles in various times with the excitation frequency function of 0.5

Radial stresses caused by Fourier and non-Fourier temperature profiles have been plotted in Figs. 4-6. Careful observation of these diagrams reveals that radial stresses under non-Fourier conditions, have a higher range of oscillations, compared with the Fourier ones. Thus, the maximum level of stress created in the cylinder under non-Fourier condition is sometimes higher than the ones Fourier ones.

With increasing the frequency of boundary temperature, the oscillation of radial thermal stresses can be seen in the cylinder according to non-Fourier temperature profiles due to thermal inertia, oscillations of thermal stresses caused by non-Fourier profiles occur in a more limited range.

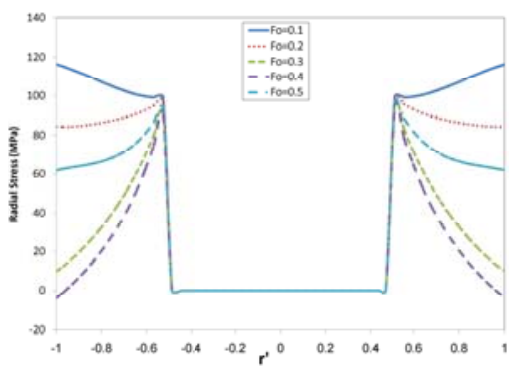

Fig. 4. Diagram of Fourier radial stress in various timings with excitation frequency function of 0.5

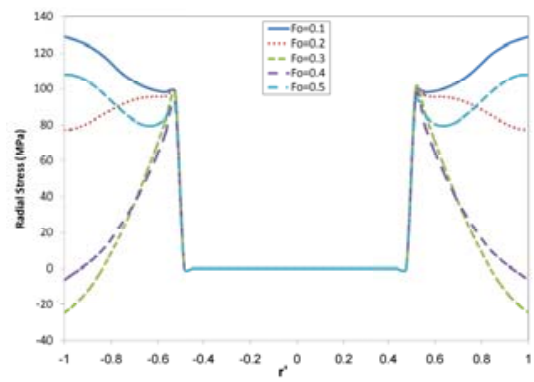

Fig. 5. Diagram of non- Fourier radial stress in various timings with excitation frequency function of 0.1

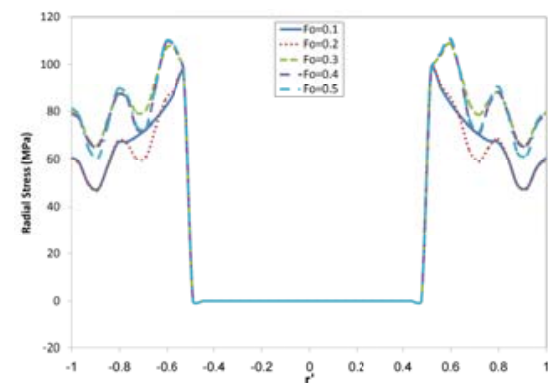

Fig. 6. Diagram of non-Fourier radial stress in various timings with excitation frequency function of 0.5

Hoop stresses have been shown in Figs. 7-9. Careful observation of these Figures reveals that the difference between Fourier and non-Fourier stresses are much more significant and the maximum hoop stress is produced under non-Fourier conditions with the excitation frequency of 0.1 which is sometimes around 5 times bigger than the Fourier one. Once again, it is observed that the increase of frequency excitation function causes limitation of the range of thermal stresses oscillations. As stated 
before, the reason for such a phenomenon is the thermal inertia of Non-Fourier temperature profiles against the imposed external changes.

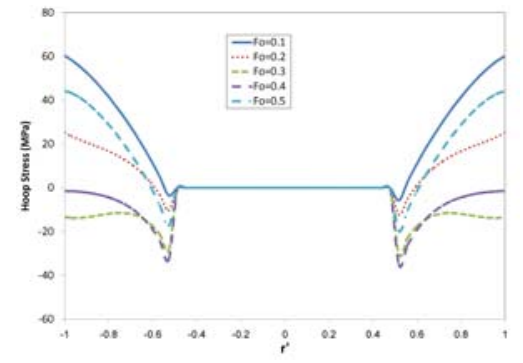

Fig. 7. Hoop stresses under Fourier condition in various timings with excitation frequency function of 0.1

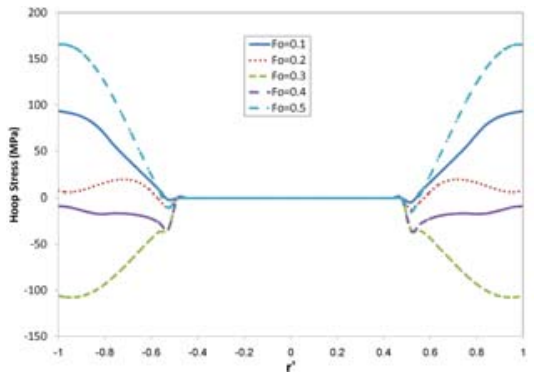

Fig. 8. Hoop stresses under non-Fourier condition in various timings with excitation frequency function of 0.1

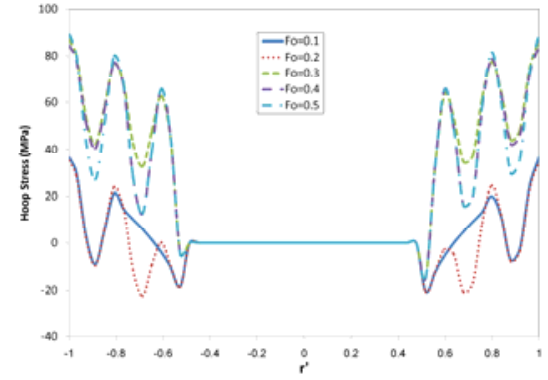

Fig. 9.Hoop stresses under nonFourier condition in various timings with excitation frequency function of 0.5

\section{Conclusion}

Analytical response of plane thermal stresses in the two Fourier and non-Fourier conditions were calculated for a long cylinder and were compared together. This solution could be used as a source of affirmation of numerical solutions of temperature profile and stress within the cylinder. Based on the diagrams the following general outcomes are observed:

- Wavy behavior of stresses and higher level of oscillation amplitude under Non-Fourier condition compared to Fourier condition

- Oscillatory convergence of transient non-Fourier profiles to the stable in comparison to rapid convergence under Fourier condition

- $\quad$ Collision and return of the thermal wave to and from the walls in non-Fourier profiles

- $\quad$ Reduction of range of fluctuations with the increase of excitation frequency function due to inertial behavior of non-Fourier temperature profile

\section{References}

Atefi, G., \& Talaee, M. R. (2011). Non-fourier temperature field in a solid homogeneous finite hollow cylinder. Archive of Applied Mechanics, 81(5), 569-583.

Bagri, A., \& Eslami, M. R. (2007). A unified generalized thermoelasticity; solution for cylinders and spheres. International Journal of Mechanical Sciences, 49(12), 1325-1335.

Cattaneo, C. (1958). A form of heat conduction equation which eliminates the paradox of instantaneous propagation. Compte Rendus, 247(4), 431-433.

Chandrasekharaiah, D. S. (1996). One-dimensional wave propagation in the linear theory of thermoelasticity without energy dissipation. Journal of Thermal Stresses, 19(8), 695-710.

Chandrasekharaiah, D. S. (1997). Complete solutions in the theory of thermoelasticity without energy dissipation. Mechanics Research Communications, 24(6), 625-630.

Chandrasekharaiah, D. S. (1998). Hyperbolic thermoelasticity: a review of recent literature. Applied Mechanics Reviews, 51(12), 705-729.

Chen, H. T., \& Lin, H. J. (1995). Study of transient coupled thermoelastic problems with relaxation times. Journal of applied mechanics, 62(1), 208-215.

Hosseini-Tehrani, P., \& Eslami, M. R. (2003). Boundary element analysis of finite domains under thermal and mechanical shock with the Lord-Shulman theory. The Journal of Strain Analysis for Engineering Design, 38(1), 53-64.

Nabavi, S. M., \& Shahani, A. R. (2009). Thermal stress intensity factors for a cracked cylinder under transient thermal loading. International Journal of Pressure Vessels and Piping, 86(2), 153-163. 
Nayfeh, A. H. (1977). Propagation of thermoelastic disturbances in non-Fourier solids. AIAA Journal, 15(7), 957-960.

Radu, V., Taylor, N., \& Paffumi, E. (2008). Development of new analytical solutions for elastic thermal stress components in a hollow cylinder under sinusoidal transient thermal loading. International Journal of Pressure Vessels and Piping, 85(12), 885-893.

Shahani, A. R., \& Nabavi, S. M. (2007). Analytical solution of the quasi-static thermoelasticity problem in a pressurized thick-walled cylinder subjected to transient thermal loading. Applied mathematical modelling, 31(9), 1807-1818.

Shen, W., \& Han, S. (2002). Hyperbolic heat conduction in composite materials. tc, 2, 0.

Taheri, H., Fariborz, S., \& Eslami, M. R. (2004). Thermoelasticity solution of a layer using the Green-Naghdi model. Journal of Thermal Stresses, 27(9), 795-809.

Talaee, M. R., \& Atefi, G. (2011). Non-Fourier heat conduction in a finite hollow cylinder with periodic surface heat flux. Archive of Applied Mechanics, 81(12), 1793-1806.

Tehrani, P. H., \& Eslami, M. R. (2000). Boundary element analysis of coupled thermoelasticity with relaxation times in finite domain. AIAA journal, 38(3), 534-541.

Vernotte, P. (1958). Les paradoxes de la théorie continue de léquation de la chaleur. Comptes Rendus Hebdomadaires Des Seances De L Academie Des Sciences, 246(22), 3154-3155.

\section{List of abbreviations}

\begin{tabular}{lclc} 
Temperature & $\boldsymbol{\theta}(\boldsymbol{k})$ & Dimensionless radius & $\boldsymbol{r}^{\prime}(-)$ \\
$\begin{array}{l}\text { Dimensionless } \\
\text { temperature }\end{array}$ & $\boldsymbol{\Theta}(-)$ & Thermal capacity & $\boldsymbol{c}(\boldsymbol{J} / \boldsymbol{k})$ \\
Time & $\boldsymbol{t}(\boldsymbol{S e c})$ & Fourier number & $\boldsymbol{F o}(-)$ \\
Flux inertia time & $\boldsymbol{\tau}_{\mathbf{0}}(\boldsymbol{S e c})$ & Vernotte number & $\boldsymbol{V} \boldsymbol{e}(-)$ \\
Thermal flux & $\boldsymbol{q}\left(\boldsymbol{W} / \boldsymbol{m}^{2}\right)$ & Frequency & $\boldsymbol{f}(\boldsymbol{l} / \boldsymbol{s})$ \\
Penetration coefficient & $\boldsymbol{\alpha}\left(\boldsymbol{m}^{2} / \boldsymbol{s}\right)$ & Stress & $\boldsymbol{\sigma}(\boldsymbol{P a})$ \\
$\begin{array}{l}\text { Thermal conduction } \\
\text { coeff. }\end{array}$ & $\boldsymbol{k}(\boldsymbol{W} / \boldsymbol{m} \boldsymbol{k})$ & Strain & $\boldsymbol{\varepsilon}(\boldsymbol{m})$ \\
Radius & $\boldsymbol{m}(\boldsymbol{r})$ & Displacement vector & $\boldsymbol{u}(\boldsymbol{m})$ \\
Elasticity module & $\boldsymbol{E}(\boldsymbol{P a} / \boldsymbol{m})$ & Poisson ratio & $\boldsymbol{v}(-)$ \\
\hline
\end{tabular}

